\section{Validação do peso e altura referidos para o diagnóstico do estado nutricional em uma população de adultos no Sul do Brasil}

\author{
Weight and height validation for diagnosis \\ of adult nutritional status in southern Brazil
}

\footnotetext{
1 Programa de

Pós-graduação em

Epidemiologia,

Universidade Federal

de Pelotas, Pelotas, Brasil.

2 Faculdade de Medicina,

Universidade Federal

de Pelotas, Pelotas, Brasil.

Correspondência

E. A. Silveira

Rua Corcovado 174,

apto. 202B, Uberlândia, $M G$

38411-092, Brasil.

erikasil@terra.com.br
}

\begin{abstract}
This study evaluated the accuracy of body mass index (BMI) based on self-reported weight and height for predicting adult nutritional status. In a cross-sectional study of 3,934 adults (> 20 years) in Pelotas, Rio Grande do Sul, Brazil, a sub-sample of 140 individuals was drawn and weight and height were measured. From the comparison between "measured" and "reported" $B M I$, the average reported BMI error was est $i$ mated and the associated factors were identified. Regardless of nutritional status, women underestimated their "reported" BMI, while in men this information was accurate. Among women, age and income were associated with underestimated BMI in a multivariate analysis. Thus, women over 50 and with lower income underestimated BMI by more than $2 \mathrm{~kg} / \mathrm{m}^{2}$. The use of "reported" BMI to predict adult nutritional status can underestimate prevalence of obesity and overestimate that of overweight in women. Correction minimizes this kind of bias, thereby making the data more accurate.
\end{abstract}

Body Mass Index; Nutritional Status; Bias (Epidemiology)
Erika Aparecida da Silveira 1

Cora Luíza Araújo 1

Denise Petrucci Gigante 1

Aluisio J. D. Barros 1

Maurício Silva de Lima 2

\section{Introdução}

Em estudos epidemiológicos, especialmente naqueles que se referem à obesidade, há necessidade de classificar o estado nutricional. Um dos critérios mais utilizados atualmente para calcular a prevalência de obesidade é o Índice de Massa Corporal (IMC) 1. Para isso, necessita-se de informações sobre o peso e a altura de cada indivíduo. Tais informações devem ser obtidas, preferencialmente, com base na mensuração direta dos indivíduos, utilizando-se para isso balanças e estadiômetros. A coleta dessas medidas, entretanto, implica diretamente custos, tempo de execução, treinamento de antropometristas, além de dificuldades de transporte desse material em trabalhos de campo.

Em países desenvolvidos, tem sido observada a freqüente utilização de peso e altura auto-referidos em estudos epidemiológicos, tanto em questionários administrados, autopreenchidos, quanto em entrevistas por telefone 2,3,4. A utilização do peso e altura referidos é uma prática pouco freqüente no Brasil, possivelmente devido à carência de estudos nacionais e de base populacional analisando a validade de tais informações 5,6.

Em outros países, o estudo da validade do peso e da altura auto-referidos mostrou alta correlação entre os valores mensurados e aqueles auto-informados; porém, alguns desses mostraram tendência a subestimativa do peso e su- 
perestimativa da altura 2,4,7,8. Esse tipo de erro poderia afetar o IMC e conseqüentemente as prevalências de sobrepeso e obesidade. Portanto, a utilização do IMC valendo-se do peso e altura referidos para predizer o estado nutricional de grupos populacionais apresentaria problemas em relação a sua validade. Além disso, a determinação dos possíveis erros de classificação e as características dos indivíduos que levam a esse tipo de erro necessitam ser mais exploradas 2,3,4,6,8.

Este estudo foi desenhado para avaliar a comparabilidade do IMC obtido pelo peso e altura auto-referidos, com o IMC obtido com base no peso e na altura medidos por pessoal treinado. Buscou-se também identificar as características sócio-econômicas, demográficas e comportamentais que identificam indivíduos com maior erro entre o IMC "referido" e o medido. Procurou-se ainda avaliar se há necessidade de correção do IMC “referido" para utilização em lugar do IMC medido.

\section{Métodos}

Este estudo é parte de uma investigação que objetivou avaliar diversos aspectos de saúde da população residente na zona urbana do Município de Pelotas, Sul do Brasil. Por meio de delineamento transversal de base populacional, foram incluídos 48 setores censitários e seus respectivos domicílios, selecionados mediante processo aleatório em múltiplos estágios. Primeiramente, sortearam-se os setores e em cada um deles uma quadra, e, nessa, a esquina a partir da qual foram visitados um em cada três domicílios até completar 44 em cada setor censitário. A amostra final, de 3.934 indivíduos com idade superior a vinte anos, foi entrevistada entre outubro de 1999 e janeiro de 2000.

Para este estudo, uma subamostra foi obtida pelo sorteio de três setores em regiões periféricas da cidade e outros três em regiões centrais, dentre aqueles 48 inicialmente selecionados. Foram incluídos os adultos com idade igual ou superior a vinte anos que se encontravam nos domicílios no momento da entrevista. Esses indivíduos já haviam respondido a um questionário completo, aplicado na etapa anterior. O tempo decorrido entre as duas entrevistas nunca excedeu a sete dias.

Foram realizadas as medidas de peso e de altura em 140 adultos. Essa amostra foi suficiente para detectar, com um poder de $90,0 \%$, uma razão de prevalências de 2,0 , razão não expostos:expostos de 4:1, considerando-se obesidade como exposição ${ }^{9}$ e prevalência de $40,0 \%$ de pessoas com excesso de peso que subestimam o próprio peso em até $2 \mathrm{~kg}{ }^{10}$. Ao número de amostra calculado acrescentou-se um excedente de $30,0 \%$, pois, na falta de uma estimativa precisa do efeito do delineamento, a prática tem mostrado que este acréscimo é, na maioria das vezes, suficiente para realizar a análise ajustada.

Os instrumentos utilizados na coleta de peso e de altura foram: balança de banheiro com capacidade de $150 \mathrm{~kg}$ e precisão de $1 \mathrm{~kg}$ e estadiômetro de alumínio com precisão de $1 \mathrm{~mm}$. A balança era calibrada ao final de cada dia de coleta de dados. A coleta das medidas foi realizada por dois nutricionistas devidamente treinados de acordo com as recomendações de Lohman et al. 11.

As demais informações foram obtidas na primeira etapa por uma equipe de entrevistadoras selecionadas e treinadas que aplicaram um questionário padronizado e pré-testado. As variáveis estudadas foram: sexo, idade em anos completos, cor (branca e não-branca), situação conjugal (com ou sem companheiro), escolaridade em anos completos, renda familiar mensal (somas das rendas individuais de cada um dos moradores do domicílio), número de moradores por domicílio, satisfação com a forma do corpo (sim ou não), atitude para mudar de peso no último ano (sim ou não), peso e altura auto-referidos.

Com as variáveis coletadas na segunda etapa do estudo foram avaliados o peso e a altura medidos, além do IMC. A classificação nutricional dos indivíduos foi realizada de acordo com os critérios recomendados pela Organização Mundial da Saúde (OMS) 12: IMC $<18,5 \mathrm{~kg} / \mathrm{m}^{2}$ (Baixo peso); IMC $\geq 18,5$ e até $24,9 \mathrm{~kg} / \mathrm{m}^{2}$ (Eutrófico); IMC $\geq 25$ e até $29,9 \mathrm{~kg} / \mathrm{m}^{2}$ (Sobrepeso) e $\mathrm{IMC} \geq 30,0 \mathrm{~kg} / \mathrm{m}^{2}$ (Obeso).

Na análise univariada, considerou-se "aceitável” quando a informação de peso foi referida com erro máximo de $3,0 \%$ em relação ao peso medido e de altura quando referida com diferença de até um centímetro em relação à altura medida.

Por intermédio das variáveis peso e altura auto-referidos construiu-se o "IMC referido" pela divisão do peso (kg) pelo quadrado da altura (m). Com o peso e altura mensurados calculou-se o IMC medido, com base na mesma equação. Considerou-se "acerto" quando o "IMC referido" permanecesse na mesma categoria do IMC medido, de acordo com a classificação nutricional da OMS.

$\mathrm{Na}$ análise bivariada, avaliou-se se a média de erro de IMC (IMC referido - IMC medido) era igual a zero, em cada categoria das variá- 
veis estudadas por meio de teste para uma amostra. Posteriormente, aplicou-se o teste de Kruskal-Wallis 13 e teste t de Student para a comparação entre médias. As variáveis que apresentaram p-valor inferior a 0,2 , nos testes acima citados, foram selecionadas para a análise multivariável realizada pela regressão linear múltipla (RLM) a fim de identificar as características associadas a variações no erro de IMC. Optou-se por uso das variáveis na forma categorizada 14 , visto que, durante a análise bivariada, as médias de erros de IMC apresentaram tendência não linear em diversas variáveis. A utilização de variáveis categorizadas deve-se, ainda, ao objetivo de identificar grupos de indivíduos com maiores erros na estimativa de IMC referido.

A entrada das variáveis na regressão múltipla foi manual e "para frente", e, no primeiro nível, avaliou-se o efeito das variáveis sócioeconômicas e demográficas (renda familiar, escolaridade e idade); posteriormente, a atitude para mudar de peso e, por último, o estado nutricional. Testou-se a presença de colinearidade entre as variáveis e, após a construção do modelo final de regressão linear, fez-se um diagnóstico de adequação do modelo pelo gráfico de resíduos, gráfico normal de resíduos e teste de Shapiro-Wilk 13.

A regressão linear permitiu identificar as características dos indivíduos com maiores erros no IMC referido; desta forma, com maior probabilidade de erro na classificação para sobrepeso e obesidade. Após a identificação desses grupos, foi proposta uma correção para o IMC referido utilizando a equação de regressão linear múltipla: IMC corrigido $=\alpha+\beta_{1}$ quartil de renda $+\beta_{2}$ categoria de idade.

A concordância entre as categorias do IMC medido e referido foi avaliada pelo coeficiente kappa, sem ponderação. A concordância entre o IMC medido e o referido em sua forma original, contínua, foi realizada pelo coeficiente de correlação de concordância (coeficiente e gráficos de Lin) 15 e pela estratégia proposta por Bland \& Altman 16. O coeficiente de correlação de concordância mede a coincidência da linha de regressão dos dados com a linha de concordância perfeita (45 graus) e combina um componente de precisão (o coeficiente de correlação de Pearson) e um de acurácia. Quando o valor do coeficiente de Lin é igual a um, significa que a reta de regressão está exatamente sobre a reta de concordância perfeita. A estratégia de Bland \& Altman inclui a construção de um gráfico de concordância (média $v s$. diferença) e o cálculo do limite de concordância. Mediante esta técnica é possível avaliar visual- mente a concordância e a magnitude das diferenças para 95,0\% das observações. Avaliou-se também o IMC referido corrigido (utilizando o resultado da regressão linear) em relação ao medido.

\section{Resultados}

Peso e altura foram medidos em 140 indivíduos; houve 9 perdas $(6,4 \%)$ por falta de informação sobre peso ou altura referidos durante a primeira etapa do estudo.

As características demográficas e sócio-econômicas da amostra foram assim distribuídas: $59,0 \%$ de mulheres, $80,0 \%$ de cor branca, $72,0 \%$ viviam com companheiro, $51,0 \%$ estavam na faixa etária entre 30 e 49 anos e, 33,0\% com 50 anos ou mais, quase $18,0 \%$ com $0-4$ anos de escolaridade e $21,0 \%$ estudaram 12 anos ou mais. A renda familiar média foi de $\mathrm{R} \$ 1.048,50$. Quanto ao estado nutricional, avaliado pelo IMC referido e IMC medido, observou-se, respectivamente, que $38,9 \%$ e $38,2 \%$ dos indivíduos apresentavam sobrepeso enquanto, $15,3 \%$ e $17,6 \%$ apresentavam obesidade. Satisfação com seu corpo foi referida por $65,0 \%$ dos indivíduos, e $31,0 \%$ não tomaram nenhuma atitude para reduzir o peso no último ano.

O peso foi referido como aceitável por $64,9 \%$ das mulheres enquanto $22,1 \%$ delas o superestimaram. Entre os homens esse acerto foi de $55,6 \%$ e a superestimativa de $38,9 \%$. A altura foi superestimada por $50,0 \%$ e $67,0 \%$ de homens e mulheres, respectivamente; $43,0 \%$ e $27,0 \%$ referiram altura satisfatoriamente.

A categoria de IMC referido coincidiu com a categoria de IMC medido em 87,0\% dos indivíduos, sendo $90,0 \%$ dos homens e $86,0 \%$ das mulheres. Apenas 2,0\% dos homens foram incluídos em categorias de IMC subestimadas, enquanto, entre as mulheres, o valor foi de $13,0 \%$.

Nota-se que homens e mulheres erram diferentemente ao referirem seu peso e sua altura (Tabela1). As mulheres superestimaram a altura, em média, um centímetro a mais do que os homens. Em relação ao peso, observa-se que os homens superestimaram, em média, $1,93 \mathrm{~kg}$ vs. $0,4 \mathrm{~kg}$ entre as mulheres. Pelo coeficiente de correlação de concordância (CCC) para peso e altura, observa-se menor concordância na altura em toda a população, sobretudo entre as mulheres. Assim, a validade do IMC referido é diferente entre os sexos. As mulheres, em média, subestimaram seu IMC em aproximadamente $1 \mathrm{~kg} / \mathrm{m}^{2}$, e entre os homens esse erro foi de $0,17 \mathrm{~kg} / \mathrm{m}^{2}$. Entre os homens a distribuição do erro de IMC é bastante homogênea e em tor- 
no de zero. Já entre as mulheres percebe-se que a distribuição apresenta maior amplitude, com maior freqüência de valores negativos; logo, subestimando o IMC medido. Com base na constatação de que erro no IMC referido apresentase diferentemente entre os sexos, as análises subseqüentes foram realizadas para homens e mulheres separadamente.

Observando-se as médias de erros de IMC conforme o estado nutricional (Tabela 2), constata-se que indivíduos com sobrepeso ou com obesidade subestimam seu IMC, e essa subestimativa está positivamente associada com o aumento do IMC. Quando se analisa a média de erro de IMC nos homens, independentemente do estado nutricional, todos apresentam um IMC referido de razoável validade. Já as mulheres relatam seu peso e sua altura de forma a subestimar seu IMC em todas as categorias de estado nutricional. Observa-se que quanto maior é o IMC das mulheres maiores são as subestimavas do IMC referido.

Tabela 1

Medianas, médias e intervalos de confiança dos erros de peso, de altura, de Índice de Massa Corporal (IMC) referido e coeficiente de correlação de concordância e seu respectivo intervalo de confiança. Pelotas, Rio Grande do Sul, Brasil, 2000.

\begin{tabular}{|c|c|c|c|c|c|c|}
\hline População & Erros & Mediana & Média & IC95\% & $\mathrm{CCC}$ & IC95\% \\
\hline \multirow[t]{3}{*}{ Todos } & De peso $(\mathrm{kg})$ & 1,00 & 1,03 & $0,50-1,56$ & 0,967 & $0,953-0,976$ \\
\hline & De altura $(\mathrm{cm})$ & 2,00 & 3,00 & $2,00-3,00$ & 0,887 & $0,848-0,917$ \\
\hline & De IMC $\left(\mathrm{kg} / \mathrm{m}^{2}\right)$ & $-0,18$ & $-0,51$ & $-0,81--0,21$ & 0,912 & $0,879-0,936$ \\
\hline \multirow[t]{3}{*}{ Homens } & De peso $(\mathrm{kg})$ & 2,00 & 1,93 & $0,91-2,96$ & 0,942 & $0,904-0,966$ \\
\hline & De altura $(\mathrm{cm})$ & 1,50 & 2,00 & $1,00-2,00$ & 0,933 & $0,890-0,959$ \\
\hline & De IMC $\left(\mathrm{kg} / \mathrm{m}^{2}\right)$ & 0,26 & 0,17 & $-0,25-0,58$ & 0,914 & $0,857-0,949$ \\
\hline \multirow[t]{3}{*}{ Mulheres } & De peso $(\mathrm{kg})$ & 1,00 & 0,39 & $-0,15-0,89$ & 0,980 & $0,969-0,987$ \\
\hline & De altura $(\mathrm{cm})$ & 2,00 & 3,00 & $2,00-4,00$ & 0,708 & $0,594-0,794$ \\
\hline & De IMC $\left(\mathrm{kg} / \mathrm{m}^{2}\right)$ & $-0,43$ & $-0,99$ & $-1,37--0,60$ & 0,909 & $0,864-0,940$ \\
\hline
\end{tabular}

$\mathrm{CCC}=$ coeficiente de correlação de concordância;

IC95\% = intervalo de confiança de $95 \%$.

Tabela 2

Médias dos erros do Índice de Massa Corporal (IMC) referido conforme o estado nutricional de toda a amostra, homens e mulheres. Pelotas, Rio Grande do Sul, Brasil, 2000.

\begin{tabular}{|c|c|c|c|c|c|}
\hline População & Estado nutricional & $\mathbf{n}$ & Médias de erros & IC95\% & p-valor \\
\hline \multirow[t]{3}{*}{ Todos } & Eutrófico & 58 & $-0,10$ & $-0,53-0,32$ & 0,63 \\
\hline & Sobrepeso & 50 & $-0,54$ & $-0,96--0,13$ & 0,01 \\
\hline & Obeso & 23 & $-1,46$ & $-2,34--0,57$ & 0,002 \\
\hline \multirow[t]{3}{*}{ Homens } & Eutrófico* & 26 & 0,42 & $-0,31-1,14$ & 0,24 \\
\hline & Sobrepeso & 22 & $-0,10$ & $-0,60-0,40$ & 0,68 \\
\hline & Obeso & 6 & 0,06 & $-1,58-1,71$ & 0,92 \\
\hline \multirow[t]{3}{*}{ Mulheres } & Eutrófico & 32 & $-0,53$ & $-1,03--0,03$ & 0,04 \\
\hline & Sobrepeso & 28 & $-0,90$ & $-1,52--0,27$ & 0,006 \\
\hline & Obeso & 17 & $-1,99$ & $-3,00--0,99$ & $<0,001$ \\
\hline
\end{tabular}

* Inclui 3 indivíduos com baixo peso:

IC95\% = intervalo de confiança de $95 \%$. 
A concordância entre as categorias do IMC medido e do referido mostrou valores de kappa de 0,86 para os homens e 0,83 para as mulheres.

Entre os homens, verificou-se que as prevalências de obesidade calculadas pelo IMC referido e o IMC medido foram iguais $(11,0 \%)$. Para sobrepeso, as prevalências foram de $41,0 \% \mathrm{e}$ $46,0 \%$, respectivamente.

A análise bivariada para o sexo masculino mostrou que somente a renda familiar acima de dez salários mínimos, média 0,43 (IC95\%: 0,03-0,83), e a insatisfação corporal, média 0,83 (IC95\%: 0,02-1,63), apresentaram médias de erros do IMC diferentes de zero. Ao se compararem as médias de IMC entre as categorias de cada variável, observou-se que nenhuma mostrou p-valor menor ou igual a 0,20. Assim sendo, não haveria razão para fazer a RLM no grupo dos homens.

No sexo feminino, a análise bivariada ( $\mathrm{Ta}$ bela 3) mostrou erros médios de IMC diferentes de zero nas seguintes categorias de variáveis: idades entre 20 e 29 anos e acima dos 50 anos; indivíduos de cor branca; indivíduos que vivem com ou sem companheiro; os três primeiros quartis de renda familiar; todos os níveis de escolaridade, mas para 12 anos ou mais se mostrou no limiar da significância; satisfação e insatisfação corporal; não ter atitude para perder peso no último ano e todas as categorias de estado nutricional (IMC medido). As características descritas acima se revelaram, então, marcadores de risco para erro de IMC referido. À medida que aumentam os anos de estudo, a qualidade do IMC referido melhora, no entanto, as médias de erros de IMC nas categorias de escolaridade mostraram-se semelhantes. Em relação ao estado nutricional, o erro médio aumenta significativamente à proporção que aumenta o IMC, observando-se uma tendência linear.

Na Tabela 3, pode-se observar também as variáveis que se mostraram associadas com erros de IMC: idade, renda familiar, atitude para perder peso no último ano e estado nutricional. Essas variáveis foram selecionadas para a análise multivariada, bem como escolaridade, por ter um p-valor menor do que 0,20.

$\mathrm{Na}$ análise multivariada para o sexo feminino, apenas idade e renda familiar permaneceram associadas ao erro de IMC. O modelo final resultou em um coeficiente de determinação (R2) de 0,24, ou seja, idade e renda explicaram $24,0 \%$ da variabilidade do erro médio de IMC ( $\mathrm{p}<0,001)$. Na tabela 4, são apresentados os valores dos erros de IMC obtidos por intermédio da equação de regressão. Com base nesses valores foi possível calcular o IMC corrigido, de acordo com faixa etária e renda familiar das mulheres.

As mulheres acima de cinqüenta anos e que se encontram nos dois quartis de menor renda familiar subestimaram o IMC referido em mais de $2 \mathrm{~kg} / \mathrm{m}^{2}$ (Tabela 4). Portanto, observa-se um erro sistemático no IMC referido, resultando na subestimativa do IMC medido.

Observando os limites de concordância nos gráficos de Bland \& Altman (Figura 1), o IMC corrigido para as mulheres melhora a precisão. Os limites de concordância entre o IMC corrigido e o IMC medido ficam distantes $2,8 \mathrm{~kg} / \mathrm{m}^{2}$ da média, enquanto, entre o IMC referido e o medido essa distância é de $3,4 \mathrm{~kg} / \mathrm{m}^{2}$. Tais valores mostram que se deve considerar um erro máximo de até $2,8 \mathrm{~kg} / \mathrm{m}^{2}$ no IMC corrigido, tendo-se como referência o IMC medido. A correção do IMC referido aumentou o CCC de 0,909 para 0,956 e a reta de regressão (gráfico de Lin) ficou praticamente sobreposta à reta de concordância perfeita (linha tracejada).

Para efeito de comparação dos limites de concordância 16 e do CCC 15, plotaram-se ainda gráficos de Bland \& Altman e gráficos de Lin para os homens, bem como para toda a população usando o IMC semi-referido, ou seja, calculado pelo peso referido e pela altura medida (Figura 1). Observa-se que o maior CCC e os menores limites de concordância apresentado na Figura 1 é o do IMC semi-referido, e a reta de regressão do IMC semi-referido com base no IMC 20,0 está praticamente sobreposta à reta de concordância perfeita.

As prevalências de obesidade feminina calculadas por intermédio dos IMC referido e medido são $18,2 \%$ e $22,1 \%$, respectivamente. Com a correção do IMC, obteve-se uma prevalência de $22,4 \%$. Assim sendo, o IMC corrigido foi equivalente ao IMC medido para diagnosticar obesidade em populações, enquanto o IMC referido é uma aproximação da prevalência medida de obesidade.

\section{Discussão}

É importante destacar inicialmente que este estudo avaliou indivíduos provenientes de uma amostra de base populacional, diferindo de alguns que avaliaram peso e altura auto-referidos 6,7 , o que minimiza a possibilidade de viés de seleção.

Ao se comparar a amostra deste estudo com a população investigada na primeira etapa do trabalho, verificou-se que a distribuição das variáveis foram semelhantes, exceto as categorias de escolaridade entre 0 e 4 anos e entre 9 e 11 anos. 
Distribuição das médias de erros de Índice de Massa Corporal (IMC) referido em mulheres, e testes para avaliação de médias. Pelotas, Rio Grande do Sul, Brasil, 2000.

\begin{tabular}{|c|c|c|c|c|c|}
\hline Variável & $\mathrm{n}$ & Médias de erros & IC95\% & p-valor* & p-valor ${ }^{\star *}$ \\
\hline Idade (anos completos) & & & & & $0,005^{\star \star \star}$ \\
\hline $20-29$ & 17 & $-0,98$ & $-1,76--0,19$ & 0,020 & \\
\hline $30-39$ & 18 & $-0,12$ & $-0,66-0,41$ & 0,630 & \\
\hline $40-49$ & 20 & $-0,54$ & $-1,25-0,17$ & 0,130 & \\
\hline $50-59$ & 11 & $-2,14$ & $-3,16--1,11$ & $<0,001$ & \\
\hline $60-80$ & 10 & $-2,01$ & $-3,63--0,40$ & 0,020 & \\
\hline Cor & & & & & $0,72^{\star \star \star \star}$ \\
\hline Branca & 63 & $-1,02$ & $-1,43--0,61$ & $<0,001$ & \\
\hline Parda/preta & 14 & $-0,84$ & $-1,96-0,29$ & 0,130 & \\
\hline Situação conjugal & & & & & $0,71^{\star \star \star \star}$ \\
\hline Com companheiro & 50 & $-0,93$ & $-1,39--0,48$ & $<0,001$ & \\
\hline Sem companheiro & 27 & $-1,08$ & $-1,81--0,35$ & 0,005 & \\
\hline \multicolumn{6}{|l|}{ Renda familiar (quartis) } \\
\hline $1 \underline{1}$ & 17 & $-1,18$ & $-2,07--0,30$ & 0,010 & $0,01^{\star \star \star}$ \\
\hline 20 & 21 & $-1,75$ & $-2,64--0,87$ & $<0,001$ & \\
\hline 3은 & 21 & $-0,83$ & $-1,52--0,14$ & 0,020 & \\
\hline 4으 & 17 & 0,08 & $-0,31-0,48$ & 0,650 & \\
\hline \multicolumn{6}{|c|}{ Escolaridade (anos completos) } \\
\hline $0-4$ & 15 & $-2,21$ & $-3,42--0,99$ & 0,001 & $0,07^{\star \star \star}$ \\
\hline $5-8$ & 18 & $-0,95$ & $-1,82--0,08$ & 0,030 & \\
\hline $9-11$ & 27 & $-0,55$ & $-1,06--0,05$ & 0,030 & \\
\hline 12 ou mais & 17 & $-0,63$ & $-1,25--0,006$ & 0,050 & \\
\hline \multicolumn{6}{|l|}{ Satisfação corporal } \\
\hline Sim & 37 & $-1,01$ & $-1,55--0,47$ & $<0,001$ & $0,91^{\star \star \star \star *}$ \\
\hline Não & 40 & $-0,96$ & $-1,52--0,40$ & 0,001 & \\
\hline \multicolumn{6}{|c|}{ Atitude para mudar de peso } \\
\hline $\operatorname{Sim}$ & 33 & $-0,52$ & $-1,05-0,003$ & 0,050 & $0,03^{\star \star \star \star}$ \\
\hline Não & 44 & $-1,33$ & $-1,87--0,80$ & $<0,001$ & \\
\hline \multicolumn{6}{|l|}{ Estado nutricional } \\
\hline Eutrófico & 32 & $-0,53$ & $-1,03--0,02$ & 0,040 & $0,02^{\star \star \star}$ \\
\hline Sobrepeso & 28 & $-0,90$ & $-1,52--0,27$ & 0,006 & \\
\hline Obeso & 17 & $-1,99$ & $-2,99--0,99$ & $<0,001$ & \\
\hline
\end{tabular}

* Teste t comparando as diferenças com valor zero (fixo):

** Teste para comparação de médias:

$\star \star \star$ Teste Kruskal-Wallis:

$\star \star \star \star$ Teste $\mathrm{t}$ de Student;

IC95\% = intervalo de confiança de $95 \%$. 
Uma possível limitação identificada referese à confiabilidade da medida de peso, uma vez que a balança utilizada tem precisão de $1 \mathrm{~kg}$. Contudo, por serem indivíduos adultos, é pouco provável que tal precisão possa influenciar a ponto de alterar as conclusões. Em razão da imprecisão da balança, considerou-se aceitável o peso referido com uma diferença de até $3,0 \%$ do peso medido.

O tempo decorrido entre a visita domiciliar para a aplicação do questionário e o dia em que o peso e a altura foram mensurados poderia resultar em alguma variação. Todavia, se tal variação ocorreu foi, provavelmente, de pequena magnitude porque esse intervalo de tempo nunca foi superior a sete dias.

As prevalências de obesidade e sobrepeso encontradas foram bastante semelhantes àquelas de outro estudo de base populacional realizado na mesma cidade em 1994 9. Nota-se apenas uma diminuição de 3,0\% na prevalência de obesidade. No entanto, os resultados de outro estudo transversal realizado em Pelotas em 1999 (não publicado) com adultos de 20 a 69 anos diferiram em aproximadamente cinco pontos percentuais das prevalências encontradas, sendo o sobrepeso superestimado e a obesidade subestimada.

Outros estudos já publicados sobre o tema diferem quanto ao delineamento, faixa etária da amostra e, especialmente em relação ao tipo de análise estatística utilizada, fato que dificulta algumas comparações com esses resultados.

$\mathrm{O}$ peso foi satisfatoriamente referido por $61,0 \%$ da amostra, o que está de acordo com estudo realizado em Porto Alegre, onde $62,0 \%$ relataram seu peso com um erro inferior a $2 \mathrm{~kg}$. Os homens superestimaram sua média de peso mais que as mulheres, e, entre elas, o intervalo de confiança incluiu valores negativos. Esses resultados diferem do estudo acima citado 5 , que encontrou subestimativa de peso entre mulheres. Estudos realizados na Finlândia, na Nova Zelândia, na Inglaterra e nos Estados Unidos encontraram subestimativas de peso, tanto entre as mulheres como entre os homens. Porém, as subestimativas de maior magnitude foram entre as mulheres, salvo na Inglaterra $4,7,8,10$. Os resultados do presente estudo mostraram-se diferentes daqueles de populações de países desenvolvidos, em que homens e mulheres subestimaram o peso, enquanto, nesta população, ambos os sexos superestimaram-no. Mas outro estudo realizado no Brasil 5 encontrou resultados semelhantes quanto aos homens, os quais também superestimaram o peso.

Vale ressaltar que, enquanto a população deste estudo incluiu adultos a partir de vinte
Tabela 4

Valores médios de erros na estimativa do Índice de Massa Corporal
(IMC) medido em mulheres, por grupos de renda e idade.
Pelotas, Rio Grande do Sul, Brasil, 2000.

Idade (anos completos) por renda familiar

Erros médios na estimativa do IMC

1ㅇ quartil de renda familiar

$20-29$

$-1,23$

30-39

$-0,45$

40-49

$-0,80$

50-59

$-2,22$

60-80

$-2,09$

2o quartil de renda familiar

20-29

$-1,67$

30-39

$-0,89$

40-49

$-1,24$

50-59

$-2,66$

60-80

$-2,53$

3o quartil de renda familiar

20-29

$-0,84$

30-39

$-0,06$

40-49

$-0,41$

50-59

$-1,83$

$60-80$

$-1,70$

4o quartil de renda familiar

20-29

$-0,10$

30-39

0,68

40-49

0,33

50-59

$-1,09$

$60-80$

$-0,96$

anos, outros estudos avaliaram peso referido apenas em amostras com idade superior a trinta anos. Talvez parte das diferenças encontradas quanto à qualidade da informação do peso referido, entre esta população e populações de outros países, possam ser explicadas pelas diferenças etárias e de escolaridade.

A superestimativa de altura em ambos os sexos está de acordo com a literatura $2,3,7,8$, sendo essa levemente superior entre os homens.

Subestimativas de IMC em ambos os sexos também foram observadas em amostra de funcionários de banco, com elevado nível educacional 6, no Rio de Janeiro, entre funcionários de uma companhia de energia e gás na França $2\left(0,29 \mathrm{~kg} / \mathrm{m}^{2} \mathrm{em}\right.$ homens e $0,44 \mathrm{~kg} / \mathrm{m}^{2} \mathrm{em} \mathrm{mu}$ lheres), em um estudo populacional em adultos maiores de 35 anos na Nova Zelândia 8 e na coorte EPIC-Oxford (European Prospective Investigation into Cancer and Nutrition) ${ }^{4}$. 


\section{Figura 1}

Gráficos de concordância de Lin e coeficiente de correlação de concordância (CCC) e gráficos de Bland e Altmam com suas respectivas diferenças médias (DM) e limites de concordância de $95 \%$ (LC), utilizando IMC medido, IMC referido, IMC corrigido e IMC semicorrigido.

\section{Figura 1a}

Mulheres

IMC medido e IMC referido em Mulheres

Nota: os dados devem cobrir a linha tracejada para perfeita concordância

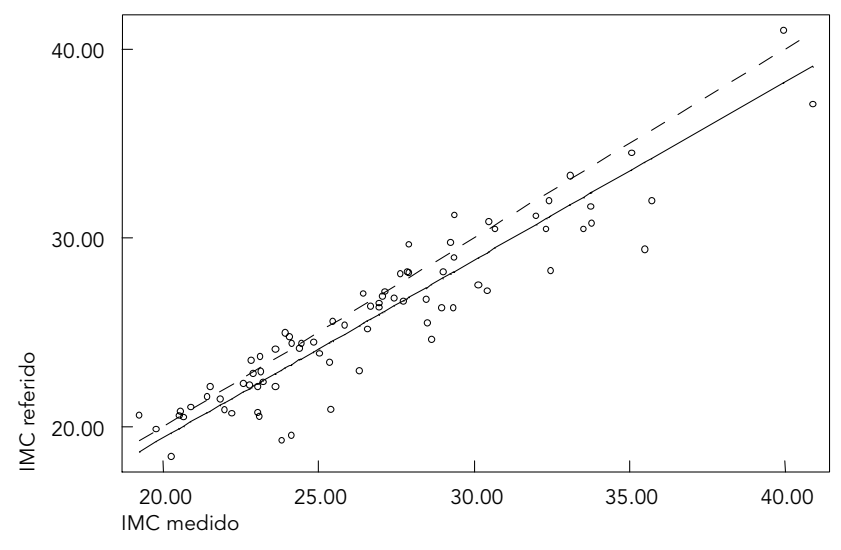

$\mathrm{CCC}=0,909$
Limites de concordância de $95 \%$

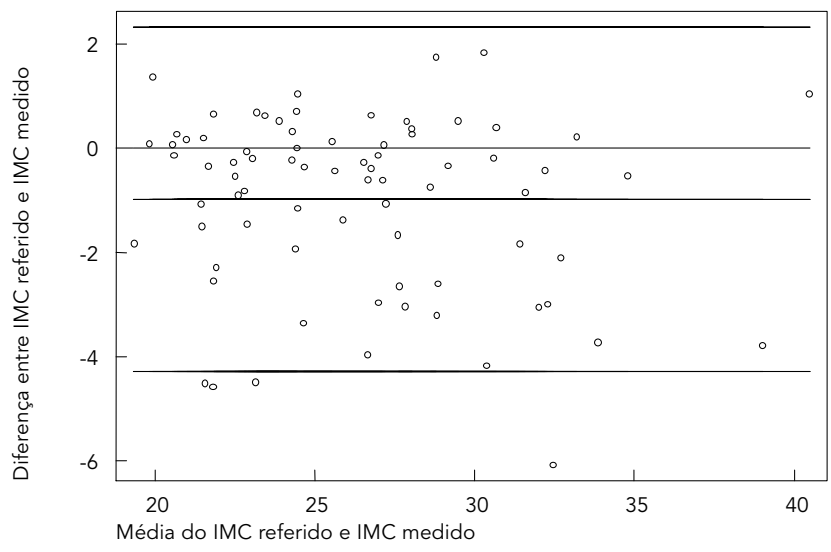

$\mathrm{DM}=0,987 \mathrm{LC}(-4,28 ; 2,31)$

IMC medido e IMC corrigido em Mulheres

Nota: os dados devem cobrir a linha tracejada para perfeita concordância

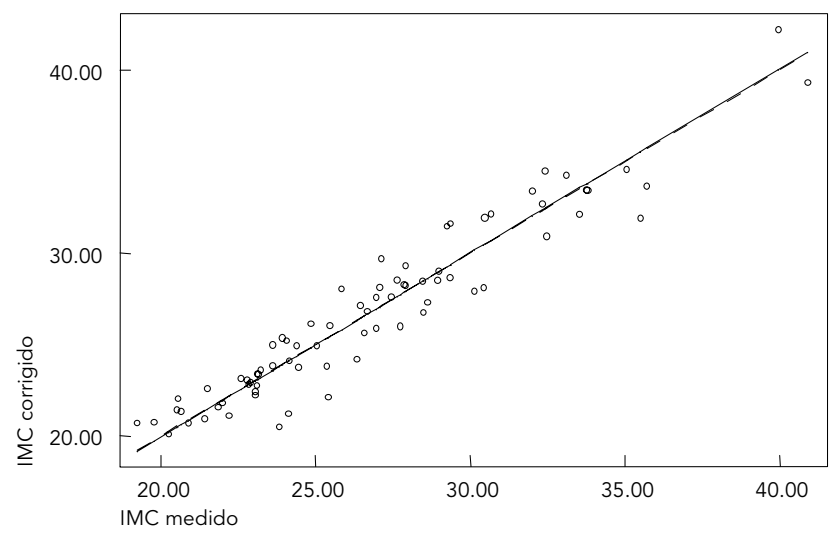

$\mathrm{CCC}=0,956$

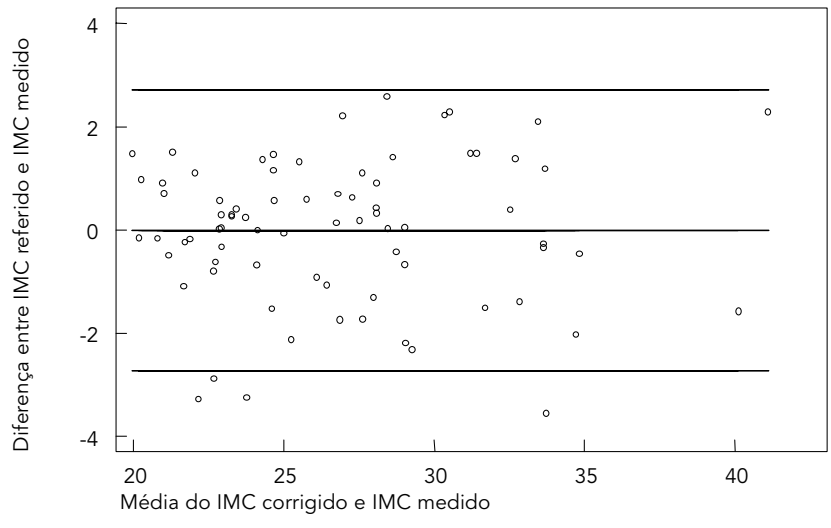

$\mathrm{DM}=-0,008 \mathrm{LC}(-2,73 ; 2,71)$

$\mathrm{IMC}=$ índice de massa corporal. 
Figura 1

Gráficos de concordância de Lin e coeficiente de correlação de concordância (CCC) e gráficos de Bland e Altmam com suas respectivas diferenças médias (DM) e limites de concordância de 95\% (LC), utilizando IMC medido, IMC referido, IMC corrigido e IMC semicorrigido.

Figura $1 b$

Homens

IMC medido e IMC referido em Homens

Nota: os dados devem cobrir a linha tracejada para perfeita concordância

Limites de concordância de $95 \%$
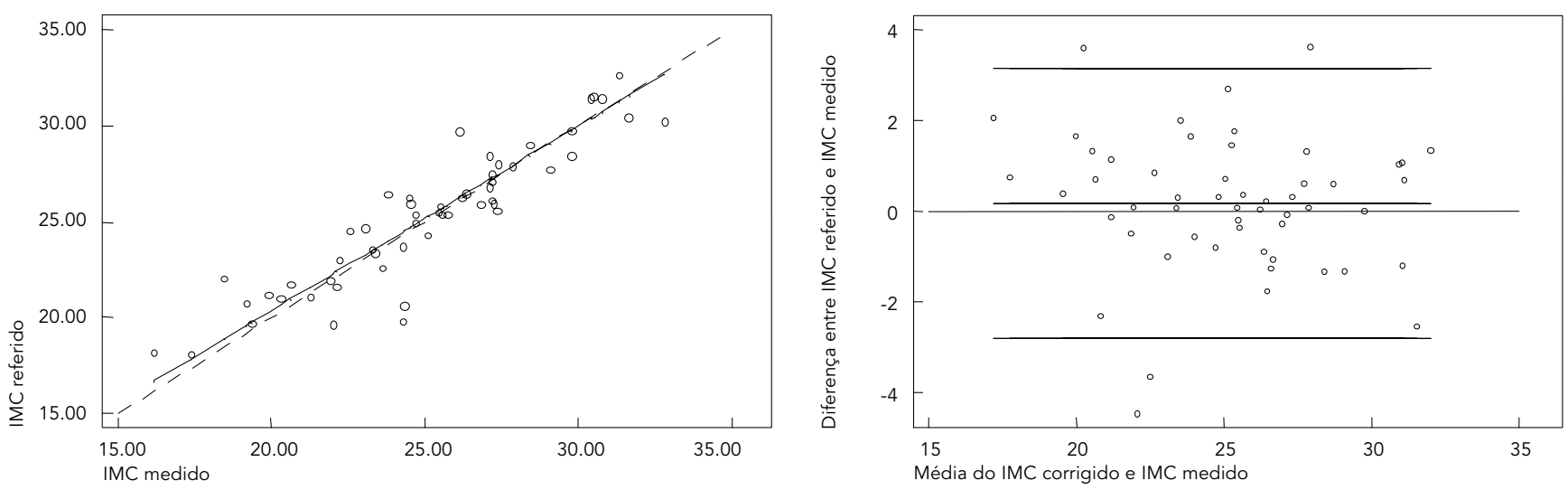

$\mathrm{CCC}=0,914$

$\mathrm{DM}=0,168 \mathrm{LC}(-2,81 ; 3,15)$

Toda a população

IMC medido e IMC semi-referido (altura medida e peso referido) em toda a população

Nota: os dados devem cobrir a linha tracejada para perfeita concordância

Limites de concordância de $95 \%$
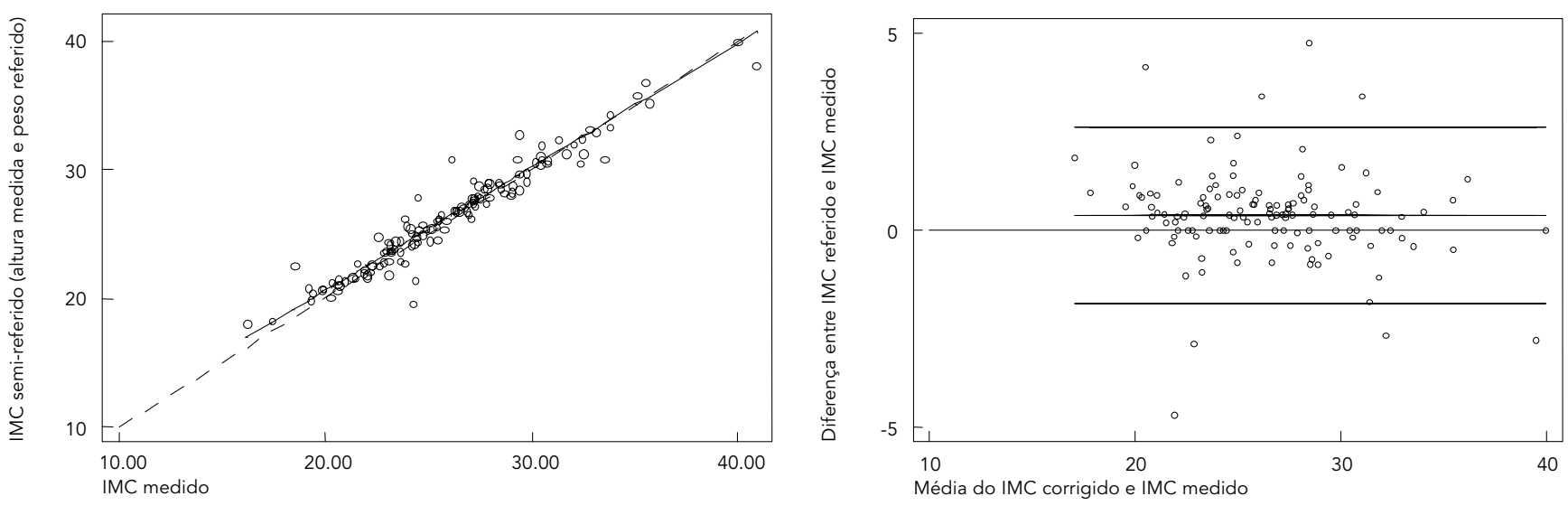

$\mathrm{CCC}=0,961$

$\mathrm{DM}=0,367 \mathrm{LC}(-1,88 ; 2,61)$

IMC = índice de massa corporal. 
Ao serem analisadas as médias de erros do IMC da população estudada, os resultados encontrados concordam com a literatura 5,8,17. Observa-se que aumentam significativamente os erros médios do IMC, conforme aumenta o IMC medido. Isto é, indivíduos com excesso de peso (sobrepeso e obesidade) relataram peso e altura de modo a subestimar o seu IMC. Porém, quando o erro médio do IMC foi estratificado por sexo, verificou-se que as subestimativas do IMC medido, relacionados ao sobrepeso e à obesidade, desaparecem em ambos os sexos. O que ocorre é que erros do IMC "referido" pelos homens não estão associados ao sobrepeso e à obesidade. Já entre as mulheres, o erro do IMC "referido" ocorre tanto entre eutróficas, como entre aquelas com sobrepeso e obesidade, sendo esse erro uma subestimativa do IMC medido. Por conseguinte, a hipótese corrente de que os indivíduos com sobrepeso e obesidade são os responsáveis pela menor validade do peso e altura informados não se confirmou. Assim, na população avaliada, a associação entre estado nutricional e menor validade do IMC "referido" pode ser atribuída à presença de fatores de confusão.

Após a análise por regressão múltipla foram detectadas as variáveis que realmente afetaram a qualidade do IMC “referido" pelas mulheres: idade e renda. Estudo realizado na Finlândia utilizando o mesmo método de análise demonstrou que entre as mulheres, idade, renda familiar e nível educacional estão positivamente associados com peso relatado incorretamente 10 . Na população de Porto Alegre 5 , somente idade e sexo mostraram-se associados com a diferença entre pesos (referido - medido) após regressão múltipla, mas renda não foi avaliada.

A relação entre os menores extratos de renda familiar e IMC "referido", de menor validade, provavelmente se deve ao menor acesso a balanças domésticas ou comerciais. Acima de cinqüenta anos de idade, isso poderia ser atribuído ao menor hábito de se pesarem e ao hábito mais raro ainda de aferirem altura. Sabese que a altura tende a diminuir com o avanço da idade 18 , o que poderia explicar, em parte, a superestimativa na altura. Dessa forma, a menor validade de peso e de altura relatados seria explicada pelo desconhecimento de seu verdadeiro peso e altura. O melhor CCC de altura observado foi nos homens, e estes, no momento da coleta de dados, quase sempre relatavam a altura da época do alistamento militar por ser a única medida de que se lembravam. Este fato reforça a hipótese de falta de informação.

A correção para o IMC "referido" aqui apresentada pode ser utilizada em outros estudos realizados em Pelotas, bem como em populações com características semelhantes.

De acordo com os aspectos analisados, conclui-se que o uso do IMC, calculado pelo peso e altura auto-referidos para avaliar o estado nutricional de grupos populacionais adultos, deve ser utilizado com cautela, pois ocorre erro de classificação levando à subestimativa da obesidade e superestimativa do sobrepeso. Esse erro na estimativa das prevalências de sobrepeso e de obesidade é mais relevante entre as mulheres de mais idade e com menores rendas. Dessa forma, a correção do IMC minimizaria esse tipo de viés, tornando os dados confiáveis. Outra opção em estudos epidemiológicos seria a utilização do peso referido com a altura medida, já que o IMC semi-referido apresentou boa validade.

Seria recomendável em estudos realizados em populações de características diferentes desta que o pesquisador utilizasse uma subamostra que permitisse identificar quais grupos de indivíduos com um IMC "referido" que poderiam levar a erros de classificação do estado nutricional (principalmente de obesidade) e, assim, realizar a correção do IMC. Com a realização de um subestudo em que peso e altura sejam mensurados numa pequena amostra, ou ainda com a coleta da medida de altura em toda a população e o uso do peso referido, podese simplificar o trabalho de campo em estudos epidemiológicos de base populacional, tornando-os mais rápidos e mais acessíveis quanto aos custos. 


\section{Resumo}

Analisou-se a validade do Índice de Massa Corporal (IMC), calculado por intermédio do peso e altura auto-referidos, para predizer o estado nutricional de adultos. Valendo-se de um estudo transversal de base populacional que inclui 3.934 indivíduos maiores de vinte anos em Pelotas, Rio Grande do Sul, Brasil, foi sorteada uma subamostra de 140 indivíduos para mensuração de peso e altura. A concordância e a validade do peso referido mostrou-se melhor do que a altura referida. Comparando-se os IMC medido e referido calculou-se o erro médio e identificaram-se os fatores associados a esse erro. O IMC "referido" é subestimado por mulheres, independentemente de seu estado nutricional; entre os homens, esse dado mostrou-se confiável. Em mulheres, idade e renda familiar mostraram-se associadas à subestimativa de IMC após análise multivariada. A utilização do IMC "referido" para predizer o estado nutricional de adultos pode resultar em subestimativa da prevalência de obesidade e superestimativa do sobrepeso em mulheres. O IMC "corrigido" calculado pela equação de regressão linear minimiza esse tipo de viés e torna os dados válidos. Outra opção é usar o peso informado com a altura medida para o cálculo do IMC.

Índice de Massa Corporal; Estado Nutricional; Viés (Epidemiologia)

\section{Colaboradores}

E. A. Silveira participou de todas as etapas da pesquisa: projeto de pesquisa, revisão de literatura, coleta de dados, elaboração do banco de dados, análise estatística e redação do artigo. C. L. Araújo participou da etapa de análise estatística e orientou a redação do artigo. D. P. Gigante participou da coleta de dados e da redação do artigo. A. J. D. Barros participou da revisão da análise estatística e da análise dos gráficos de Lin. M. S. Lima participou da coleta de dados.

\section{Referências}

1. Monteiro CA. Epidemiologia da obesidade. In: Halpern A, Matos AFG, Suplicy HL, Mancini MC, Zanella MT, organizadores. Obesidade. São Paulo: Lemos; 1998. p. 15-30.

2. Niedhammer I, Bugel I, Bonenfant S, Goldberg M, Leclerc A. Validity of self-reported weight and height in the French GAZEL cohort. Int J Obes Relat Metab Disord 2000; 24:1111-8.

3. Kuczmarski MF, Kuczmarski RJ, Najjar M. Effects of age on validity of self-reported height, weight, and body mass index: findings from the Third National Health and Nutrition Examination Survey 1988-1994. J Am Diet Assoc 2001; 101:28-34.

4. Spencer EA, Appleby PN, Davey GK, Key TJ. Validity of self-reported height and weigh in 4808 EPIC-Oxford participants. Public Health Nutr 2002; 5:561-5.

5. Schmidt MI, Duncan BB, Tavares M, Polanczyk CA, Pellanda L, Zimmer PM. Validity of self-reported weight: a study of urban Brazilian adults. Rev Saúde Pública 1993; 27:271-6.

6. Chor D, Coutinho ES, Laurenti R. Reliability of self-reported weight and height among state bank employees. Rev Saúde Pública 1999; 33:16-23.

7. Palta M, Prineas RJ, Berman R, Hannan P. Comparison of self-reported and measured height and weight. Am J Epidemiol 1982; 115:223-30.

8. Stewart AW, Jackson RT, Ford MA, Beaglehole R. Underestimation of relative weight by use of selfreported height and weight. Am J Epidemiol 1987; 125:122-6.

9. Gigante DP, Barros FC, Post CL, Olinto MT. Prevalência e fatores de risco para obesidade em adultos obesos. Rev Saúde Pública 1997; 31:236-46.

10. Jalkanen L, Tuomilehto J, Tanskanen A, Puska P. Accuracy of self-reported body weight compared to measured body weight. A population survey. Scand J Soc Med 1987; 15:191-8.

11. Lohman TG, Roche AF, Martoreli R. Anthropometric standardization reference manual. Champaign: Human Kinetics Publishers; 1988.

12. World Health Organization. Diet, nutrition, and the prevention of chronic diseases. Geneva: World Health Organization; 1990. (WHO Technical Report Series 797).

13. Altman DG. Practical statistics for medical research. London: Chapman \& Hall; 1997.

14. Kirkwood BR. Essentials of medical statistics. London: Blackwell Science; 1995.

15. Lin LI. A concordance correlation coefficient to evaluate reproducibility. Biometrics 1989; 45:255-68.

16. Bland JM, Altman DG. Comparing methods of measurement: why plotting difference against standart method is misleading. Lancet 1995; 346:1085-7.

17. Villanueva EV. The validity of self-reported weight in US adults: a population based cross-sectional study. BMC Public Health 2001; 1:11-5.

18. World Health Organization. Physical status: the use and interpretation of anthropometry. Geneva: World Health Organization; 1995. (WHO Technical Report Series 854).

Recebido em 14/Out/2003

Versão final reapresentada em $02 / \mathrm{Jul} / 2004$

Aprovado em 02/Ago/2004 